\title{
Metodología de estudio para la definición del proyecto de intervención en el retablo mayor de la Capilla Real de Granada
}

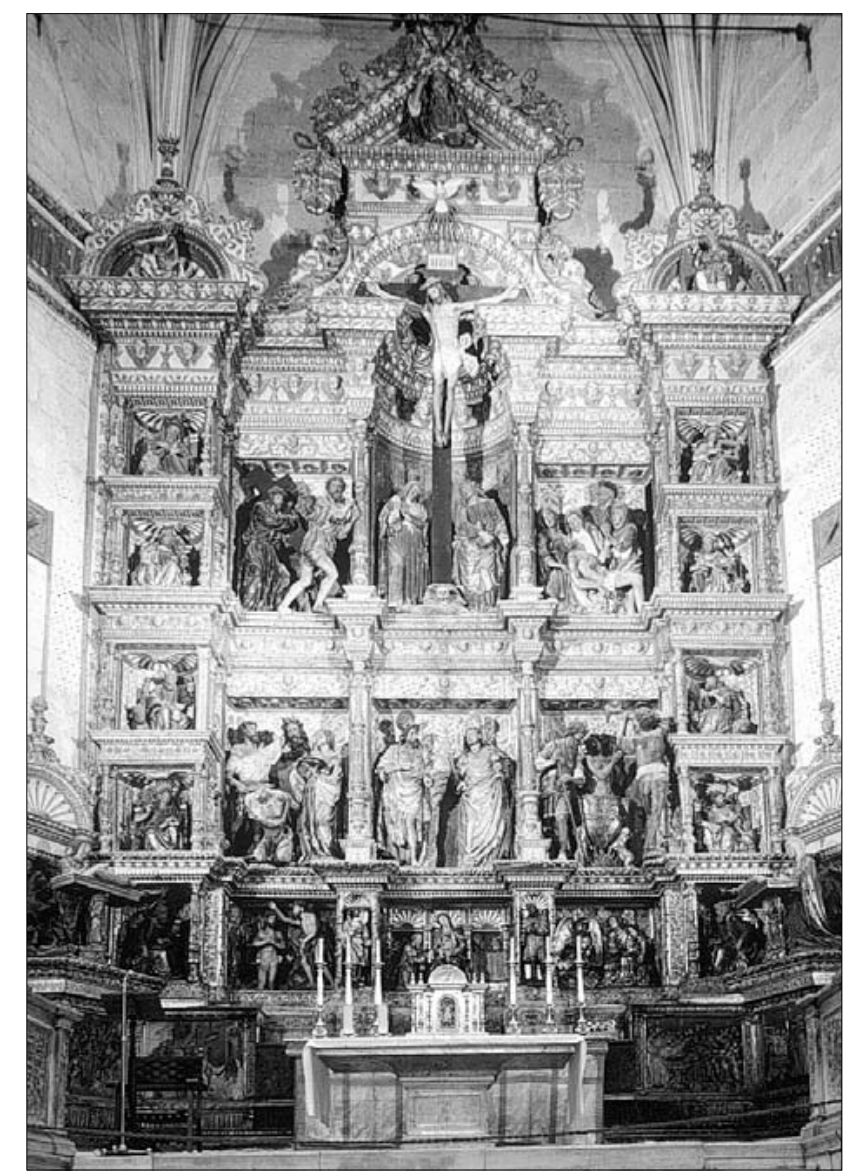

Ma José González López

Asesor técnico en conservaciónrestauración de bienes muebles del Centro de Intervención del IAPH

\section{Introducción}

El proyecto de intervención del Retablo Mayor de la Capilla Real de Granada se enmarca dentro del Convenio suscrito entre la Consejería de Cultura de la Junta de Andalucía, la Fundación Caja Madrid y el Arzobispado de Granada, firmado en la ciudad de Granada el 3 de Noviembre de 1997. Además del mencionado retablo, dicho Convenio incluye los siguientes bienes culturales: paramentos lapídeos de las zonas anexas al retablo, epigrafía y heráldica de la cabecera, así como los frontales de altares del citado retablo.

La participación del Instituto Andaluz del Patrimonio Histórico (en adelante IAPH) en este proyecto se concreta en la ejecución de los siguientes apartados:

- Definición de los contenidos.

- Realización de los estudios previos.

- Dirección y seguimiento.

- Montaje final del documento de Proyecto. 


\section{IDENTIFICACIÓN: FICHA TÉCNICA}

Municipio: Granada

Provincia: Granada

Ubicación: Presbiterio de la Capilla Real

Propietario: Arzobispado de Granada. Cabildo Capilla Real

Nombre, título: Retablo de los Santos Juanes

Atribución y/o autor:

Vigarny, Felipe. Escultor (atribuido)

Berruguete, Alonso. Escultor (atribuido)

Florentino, Jacobo. Escultor (atribuido)

Plasencia, Antón de. Pintor-decorador (atribuido)

Salamanca, Alonso. Pintor-decorador (atribuido)

Sorlózano, Andrés. Entallador (atribuido)

Bello, Martín. Entallador (atribuido)

Bernal, Miguel. Entallador (atribuido)

Cronología: 1520-1522

Tipología: Retablo

Técnica:

- Arquitectura:

Soporte: Madera

Técnica polícroma: Pintura al temple u óleo según los casos.

Técnica de decoración: Dorados bruñido y brocado aplicado según las zonas.

\section{- Esculturas:}

Soporte: Madera

Técnica polícroma: Pintura al temple u óleo según los casos.

Técnica de decoración: Dorados bruñido y brocado aplicado según las zonas.

Estilo: Renacimiento.

Escuela: Española.

Dimensiones: $9.27 \mathrm{~cm}$ de ancho $\times 16.35 \mathrm{~cm}$ de alto Inscripciones: marcas, firmas: No se observan.

\section{Metodología general aplicada para la definición de la estructura del proyecto}

Cualquier estudio e intervención de bienes culturales no se puede abordar desde la óptica meramente operativa, a ella se llega tras una fase de rigurosa investigación que permite individuar una serie de parámetros de interés aplicado al caso concreto objeto de estudio, y que posteriormente, nos permite definir la intervención aplicada a las características y necesidades del bien.

Para llegar a ello, desde la metodología de estudio e intervención que aplica el Instituto Andaluz del Patrimonio Histórico en cualquier actuación que realiza sobre bienes culturales, es necesario efectuar en primer lugar una fase cognoscitiva que incluya los diferentes estudios necesarios para individuar los factores de deterioro, las circunstancias de riesgos, las patologías presentes y los materiales y técnicas cons- titutivas, con objeto de que sus resultados nos permitan formular en una segunda fase, denominada operativa, el proyecto de actuación que requieren los bienes tomados en consideración.

Esta metodología permite establecer los criterios deontológicos y los tratamientos y materiales a emplear en cada una de las intervenciones. Así mismo, aporta los datos imprescindibles para definir el proyecto de mantenimiento y las acciones complementarias que sobre el entorno sean necesarias ejecutar con objeto de garantizar, tanto la permanencia y transmisión al futuro de los objetos intervenidos, como su presentación y disfrute por el público de la forma más correcta posible en función de las características y tipologías de los bienes.

El objetivo principal que pretendemos conseguir en cualquier actuación que se plantea desde el IAPH sobre bienes culturales es su conservación en el tiempo en las mejores condiciones posibles. Partiendo de esta premisa, y continuando con la labor que este Instituto y la administración pública andaluza está desarrollando en materia de Patrimonio Cultural, es fundamental definir un proyecto integral, siguiendo el modelo puesto a punto con éxito por el IAPH en el Proyecto Capilla Real de Granada !.

Dicho proyecto integral, aplicado al caso concreto del Retablo Mayor de la Capilla Real de Granada, intenta por un lado profundizar en las vertientes básicas del conocimiento aplicado a la intervención, siguiendo las pautas marcadas por este Instituto en esta Capilla Real y avaladas por la experiencia de otras Instituciones nacionales e internaciones que las aplican con éxito. Estas vertientes se articulan como sigue:

- Investigación.

- Acción interdisciplinar: Investigación aplicada al diagnóstico

- Definición de los criterios teórico-prácticos de intervención.

- Definición de la intervención.

- Cuantificación económica.

La investigación aplicada al conocimiento de los bienes culturales hoy día se entiende como condición previa sin la cual no tiene sentido abordar cualquier tipo de actuación. Es norma aceptada por todos parte de la siguiente premisa: la intervención nunca ha de ser ejecutada de forma arbitraria, sino que debe estar avalada por aquellos estudios necesarios que faciliten la comprensión y el conocimiento del bien cultural, ya que la actuación no resulta efectiva al cien por cien si antes no se conocen, ni se eliminan las causas que originan las alteraciones, ni se dispone de los conocimientos necesarios para poner los medios que requiere el bien cultural; no solamente aquellos que implican intervención directa sobre el bien (intervención curativa o conservativa según los casos), sino también, los que de forma indirecta requiera el entorno para garantizar su conservación y facilitar su contemplación (intervención preventiva). 
Esta concepción nos lleva necesariamente a la articulación multidisciplinar, es decir a la configuración de un equipo de trabajo interdisciplinar, de tal forma que cada especialista aporte, desde su óptica profesional aquellas informaciones de interés del bien en estudio. Informaciones complementarias entre si que van a garantizar su conocimiento, y de consecuencia, aportar resultados suficientemente avalados para definir los criterios teóricos, el índole de la intervención y su cuantificación económica.

La aplicación de esta metodología de trabajo ha permitido en el caso concreto que nos ocupa:

- Definir la estructura del proyecto.

- Satisfacer los objetivos generales y especíicos planteados.

- Definir los estudios previos necesarios para establecer el diagnóstico y la propuesta de intervención en función de las necesidades demandas por el/los bien/nes.

- Individuar los factores de alteración.

- Determinar las metodologías aplicadas a la elaboración del diagnóstico y a definir los criterios y la propuesta de intervención.

- Elaborar el proyecto de intervención más adecuado a las necesidades de los bienes.

- Cuantificar el coste de las operaciones contempladas en el Proyecto de Intervención.

\section{Estructura del proyecto}

La estructura del proyecto establecida para este bien cultural responde al siguiente esquema:

\section{Introducción}

II. Objetivos

III. Metodología general del proyecto

IV. Estudios preliminares

IV.I Levantamiento fotogramétrico.

IV.2. Estudios histórico-artísticos:

IV.3. investigación científico-analítica:

IV.4. Determinación del microclima y de los niveles de iluminación.

IV. Estado de conservación del retablo: diagnostico y factores de alteración.

V.I. Estado de conservación y diagnóstico del reverso del retablo.

V.2. Estado de conservación y diagnóstico del anverso del retablo.

V.3. Estado de conservación y diagnóstico de los paramentos y heráldica.

V.4. Estado de conservación y diagnóstico de los frontales de altar.
V.6. Agentes de alteración directos e indirectos que inciden en el actual estado de conservación del retablo.

VI. Propuesta de intervención.

VI.I. Metodología de trabajo.

VI.2. Criterios generales y específicos de intervención y mantenimiento.

VI.3. Propuesta de intervención del retablo: descripción de los tratamientos, productos y metodología de actuación en cada uno de los elementos constitutivos del retablo:

VI.3. Propuesta de intervención de los paramentos y heráldica: descripción de los tratamientos, productos y metodología de actuación en cada uno de los elementos constitutivos:

VI.4. Propuesta de intervención de los frontales de altar: descripción de los tratamientos, productos y metodología de actuación en cada uno de los elementos constitutivos

\section{Desglose presupuestario de la intervención.}

VII. I: Infraestructura básica

VII.2: Material fungible

VII.3: Personal

Su desarrollo y redacción se ha llevado a cabo con personal técnico del Instituto Andaluz del Patrimonio Histórico, en concreto con los especialistas de los Departamentos de Tratamiento y de Análisis y con el equipo de restauradores designado por la Comisión Técnica del Proyecto 2 .

\section{Objetivos}

La aplicación de esta metodología de trabajo ha permitido satisfacer los objetivos generales y específicos establecidos de antemano, concretamente:

El objetivo principal ha sido definir las investigaciones y actuaciones necesarias para establecer con garantías las intervenciones que requieren los bienes muebles en él contenido, es decir:

- Retablo Mayor

- Paramentos

- Epigrafía y heráldica

- Frontales de altar

Su definición es consecuencia directa de los resultados derivados de las investigaciones y estudios preliminares desarrollados y responde en todos su ámbitos a las necesidades demandas por ellos, es decir, a su estado de conservación, a las características técnicas y de ejecución y sobre todo, a los factores de alteración que directa e indirectamente han motivado las patologías presentes.

Partiendo de estas consideraciones previas, cabe decir también que durante su desarrollo se han marcado alcanzar una serie de objetivos secundarios que brevemente se exponen a continuación: 


\section{Levantamiento y restitución fotogramétricas escala} I:20 del retablo mayor.

La restitución fotogramétrica completa del retablo permite disponer de informaciones y material gráfico de considerable interés para el conocimiento y la comprensión del estado actual en que se encuentra el retablo (localización y distribución de las patologías presentes, datos técnicos y sistemas constructivos, etc). Informaciones todas ellas útiles, tanto para la realización del proyecto de intervención, como para documentar la intervención, al igual que para implementar los principales aspectos derivados de su estudio.

Este trabajo permite obtener:

- Dimensiones reales del retablo ya que existe un apoyo topográfico.

- Determinación exacta de la superficie del retablo.

- Visión estereoscópica del retablo.

También se ha efectuado el alzado y los perfiles del retablo con vistas a disponer de datos fiables y útiles en el momento de diseñar y montar el sistema de andamios necesario para la intervención.

Al igual que se ha realizado la Ortofoto $^{3}$ del retablo con objeto de disponer de una imagen real a color del mismo.

\section{Investigación histórico-artística:}

Este estudio se ha centrado en los siguientes aspectos:

- Realización de la historia material del retablo, basándose sobre todo el los datos encontrados en los archivos de la capilla Real y Curia Eclesiástica de Granada, también se ha consultado la bibliografía existente en las bibliotecas y hemerotecas del Museo de la Casa de los Tiros y de la Facultad de Letras de la Universidad de Granada. En este sentido, ya se disponen de datos que determinan intervenciones de reparación realizadas en diferentes épocas.

- Estudios histórico artísticos. Se ha efectuado una primera aproximación al estudio histórico- artístico del retablo, cuyos primeros resultados quedan incluidos en cada una de las fichas técnicas de las piezas que componen el conjunto del retablo, según la siguiente estructura: estructura-arquitectura, conjunto de esculturas y grupos escultóricos.

Estos estudios se han de continuar en fase de intervención con objeto de implementar a estos primeros avances los datos que se deriven del proceso de intervención. Fundamentalmente, influirá en las conclusiones finales el análisis del retablo una vez desprovisto de los depósitos superficiales y de los barnices oxidados que enmascaran sus características técnicas y estilísticas. De igual forma se deberá continuar la búsqueda de datos en archivos, en concreto en el de Simancas y en el de Zaragoza.

\section{Investigación científico-analítica:}

Se ha realizado la toma de muestras de áreas significativas. En concreto de:

- Capas polícromas (62 muestras extraídas).

- Soportes (30 muestras extraídas)

El objetivo de esta investigación ha sido determinar la naturaleza de los materiales presentes en cada uno de los estratos constitutivos tanto de los originales como de los añadidos.

En concreto este trabajo se ha centrado en:

- Obtención de la estratigrafía y determinación de las capas polícromas presentes: original y superposiciones.

- Identificación de los principales materiales constitutivos que integran cada uno de los estratos de los que está compuesta cada estratigrafía.

- Estudio de los materiales que intervienen en la realización de los brocados aplicados, con especial referencia a la masa de impresión.

\section{Documentación fotográfica:}

Comprende la documentación del estado de conservación y de la técnica de ejecución de los bienes aplicando técnicas normales y especiales (Ultravioletas).

Se ha efectuado el barrido fotográfico que documenta el estado actual del conservación y aspectos técnicos significativos del retablo. En total este trabajo ha generado 278 diapositivas en formato $35 \mathrm{~mm}$ y I general del retablo en $6 \times 6 \mathrm{~mm}$. Las técnicas empleadas han sido las siguientes: General y detalles con luz normal e iluminación rasante y detalles con iluminación ultravioleta.

\section{Exámen radiográfico y reflectográfico I.R.}

Las condiciones de trabajo y las características del andamio instalado no ha permitido efectuar este estudio "in situ" con seguridad. Es por ello que nos hemos visto obligado a posponerlos hasta la fase de intervención, una vez que las esculturas o grupos escultóricos estén desmontados del retablo y ubicados en el taller de restauración (Coro), será entonces cuando las condiciones operativas de seguridad y ámbito de trabajo permitirá realizarlos con garantías y seguridad.

\section{Determinación de microclima y de los niveles de iluminación:}

Se ha efectuado un estudio preliminar indicativo de las condiciones medioambientales y de iluminación del retablo. Este estudio se ha realizado de forma complementaria al que el IAPH efectuó durante las anualidades de 1991-1994, en relación con el proyecto de las colecciones del Museo-Sacristía de la Capilla Real. En esta ocasión se ha efectuado una comprobación de los datos microclimáticos (humedad relativa y temperatura). 
También se han determinado los niveles de la iluminación actualmente existente en el retablo midiendose los siguientes parámetros: Niveles de iluminación (lux), niveles de radiación (ultravioletas) y temperatura de color.

7. Diagnóstico: estado de conservación, determinación de factores de alteración y patologías.

Han tenido como objetivo establecer el diagnóstico que presenta los bienes incluidos en este proyecto con objeto de poder definir: los criterios de actuación, la metodología de trabajo, así como los tratamientos que requieren en base a las necesidades detectadas y también, para poder cuantificar el alcance económico de la ejecución de la intervención.

Se ha realizado la cartografía necesaria para poder representar en cada uno de sus estratos constitutivos (soporte y decoración polícroma) aquellos datos relacionados con el estado de conservación (alteraciones) y con la técnica de ejecución ( todos y cada uno de los aspectos técnicos y constructivos).

8. Evaluación de los sistemas de anclaje de la estructura del retablo al soporte murario.

El estudio de las áreas accesibles del reverso del retablo ha permitido evaluar su estado de conservación y de consecuencia, definir aquellas actuaciones que requiere con vistas a su intervención (estructura, arquitectura del retablo y anclaje al soporte murario). Este estudio se ha efectuado dentro de los límites de seguridad mínimos ya que el acceso es difícil y permite sólo la visión de una parte del mismo.

\section{Metodología aplicada para definir el estado de conservacón del retablo: Diagnóstico y factores de alteración}

Conocer para intervenir es la propuesta básica de la metodología de la intervención sobre el Patrimonio Histórico-Artístico por la que opta el IAPH en sus actuaciones. Conocimiento que debe entenderse en un sentido amplio, es decir, englobando todas las perspectiva de estudio que ofrezca un determinado bien cultural. Desde nuestra perspectiva es básico en este proyecto partir del conocimiento previo de la obra; conocimiento del cual se va a derivar posteriormente tanto el contenido como el alcance de la intervención propiamente dicha.

El estudio de un obra tan monumental y compleja como las que nos ocupa ha exigido la instalación de un sistema de andamios que permita acceder o, aproximarnos lo más posible, a todos y a cada uno de los elementos constitutivos del retablo. Se ha efectuado con las limitaciones propias derivadas de un trabajo "in situ" y de un sistema de andamios provisional cuyo montaje ha obedecido únicamente a fines de estudio. Es por ello que no se han desplazado ni movido las piezas de su disposición original.
La metodología empleada para alcanzar los fines perseguidos parte de la realización de los siguientes investigaciones:

Sistemas constructivos y técnica de ejecución. Abordar el estudio de un retablo no siempre es posible. Los resultados que se derivan de este análisis exhaustivos aportan datos, la mayoría de las veces desconocidos, que sirven de gran ayuda a todos los especialistas implicados en el desarrollo del proyecto. Es una ocasión única para conocer su estructura y sistema constructivo, por lo general raramente descrita debido a las dificultades de acceso; también es importante para determinar las técnicas polícromas y decorativas que intervienen en su decoración, que como en este caso son muy variadas y complejas, algunas de ellas, como la técnica de dorado denominada "brocado aplicado", es poco conocida y está escasamente documentada y estudiada en nuestra región andaluza.

Patologías presentes. Detectar las patologías presentes, con especial referencia a su tipología, incidencia, distribución y localización, tanto de las alteraciones derivadas del propio envejecimiento y evolución del retablo, como de las producidas por intervenciones anteriores.

Factores de deterioro. Determinar los principales agentes de alteración que han provocado directa o indirectamente los deteriores existentes, en relación con su entorno y con los derivados de la propia evolución del bien.

Estado de conservación. Realizar el diagnóstico general y específico de los bienes a partir de la inspección ocular exhaustiva que pone de manifiesto las características técnicas y constructivas y las patologías presentes. Esta primera aproximación se confirma con diferentes técnicas de apoyo, como son el examen con luz rasante, con instrumentos de aumentos, o con iluminación ultravioleta (como ya se ha comentado las características de las obras y del andamio no han permitido efectuar el estudio radiográfico de las esculturas, por los que este estudio se pospone a la fase de intervención).

A este diagnóstico se implementan por un lado, los resultados de las investigaciones analíticas efectuadas sobre las muestras extraídas de áreas significativas, previamente seleccionadas en relación con los datos o hipótesis a confirmar que permiten precisar los materiales constitutivos presentes (pigmentos, aglutinantes, adhesivos, protectivos, etc), tanto de los originales, como de los posibles añadidos, y por otro, aquellos datos derivados del estudio histórico del bien, en concreto aquellos aportados por la historia material, que permiten establecer las coincidencias entre las intervenciones o alteraciones detectadas con las descritas en las distintas fuentes documentales.

Todos estos datos nos permitirán definir el estado de conservación general y específico de cada bien, así como con vistas a la planificación de la intervención, su clasificación y prioridad en base a su urgencia. 
La metodología de estudio descrita se ha centrado básicamente en:

I. Codificación de cada uno de los elementos constitutivos. Este código ha sido empleado por todos los técnicos y especialistas implicados en el proyecto (historiadores, restauradores, fotógrafo, químicos, etc) con objeto de unificar criterios.

2. Documentación gráfica y fotográfica de aquellos aspectos más significativos y representativos tanto desde el punto de vista técnico y constructivo, como desde el punto de vista conservativo

3. Registro y clasificación de la documentación fotográfica generada. Para poder controlar y sistematizar la documentación producida.

4. Realización de la cartografía del retablo y de sus elementos. Dicha cartografía servirá de base a la representación gráfica de los siguientes aspectos de interés: datos técnicos, alteraciones e intervenciones anteriores en cada uno de los estratos constitutivos:

- Estructura arquitectónica del retablo

- Soporte (lignario/lapídeo).

- Decoración/policromía.

5. Catálogo de los motivos decorativos presentes en los estofados y brocado aplicado de la decoración de la arquitectura del retablo y de las esculturas que lo componen con reproducción del módulo a escala real.

6. Primera aproximación al estudio de superposiciones policromas presentes. Las condiciones de trabajo y el grado de actual de acumulaciones y depósitos superficiales presentes en el retablo en su conjunto, sólo han permitido efectuar un estudio preliminar de las superposiciones polícromas, debiendose completar este estudio en fase de intervención cuando las obras estén ubicadas en el Coro. No obstante los datos disponibles en la actualidad permiten establecer con claridad las áreas originales y las superpuestas por repolicromías de cada uno de los elementos y esculturas que lo componen.

7. Se han realizado aquellas pruebas que permiten poner a punto los tratamientos más comprometidos en curso de intervención. En concreto se han efectuado las siguientes:

Metodología de limpieza de la superficie policroma / decorativa: test de solubilidad, realización de catas y determinación de los diferentes niveles de limpieza.

Estas catas se han efectuado en distintas áreas del retablo, en concreto en aquellas zonas cuya composición material o técnica de ejecución, hacía necesaria la puesta a punto de una metodología de trabajo específica con vistas a definir la intervención: Dorados, brocado aplicado, rojos, corladuras, carnaciones y estofados principalmente.

Estas catas se pueden apreciar directamente en el retablo ya que se han realizado en lugares visibles desde el nivel del pavimento.
8. Realización del diagnóstico del estado de conservación de los elementos escultóricos y de la arquitectura del retablo. Se han efectuado los informes técnicos respectivos que incluyen las características técnicas y constructivas así como el estado de conservación de cada uno de los elementos del retablo. Se ha estructurado como sigue:

- Estructura-arquitectura.

- Grupos escultóricos.

- Esculturas exentas.

- Paneles.

En cada uno de los respectivos informes se incluyen los siguientes aspectos:

- Ficha técnica.

- Estudios históricos.

- Datos técnicos y estado de conservación del soporte.

- Datos técnicos y estado de conservación de la capa polícroma, dorado y capa de protección.

\section{Metodología aplicada para establecer los criterios y la propuesta de intervención}

\section{Criterios gererales de intervención}

Los criterios de intervención tomados en consideración están basados en las necesidades que demanda la propia obra, ya que es ella quien que condiciona los criterios específicos a adoptar. Si bien nos basamos en las directrices aceptadas internacionalmente para el estudio e intervención de bienes que como los que estudiamos en este proyecto tienen un elevado interés histórico y artístico. En concreto en las Cartas del Restauro de 1972 y 1987 y más recientemente en el Código Deontológico de E.C.C.O. ${ }^{4}$

Partimos de la premisa, que cualquier intervención en una obra de arte, con independencia de su tipología, debe realizarse partiendo del mayor respeto hacia la obra. Esta regla se complementa con unos conceptos básicos y generales a aplicar en cualquier intervención sobre bienes culturales y que se expone resumidamente a continuación:

- Necesidad de la intervención. La actuación ha de estar justificada por el estado de conservación y nunca debe responder a satisfacer meros principios estéticos.

- La importancia de la interdisciplinariedad y del trabajo en equipo de todos los especialistas que, directa e indirectamente, intervienen, estudian e investigan el bien cultural. Esta metodología está encaminada no sólo a establecer una diagnosis de la obra, sino también a valorar la propia metodología de intervención y a garantizar la validez de la actuación.

- Detectar y eliminar previamente a la intervención los factores de deterioro que directa e indirectamente han incidido en el estado de conservación 
del bien, potenciando o desarrollando la aparición de alteraciones en él.

- La intervención mínima. Los tratamientos deben reducirse a los que estrictamente demande la obra en cuestión.

- La intervención ha de respetar la doble polaridad que plantea una obra de arte: La actuación sobre el aspecto histórico y estética, y la acción directa sobre su materia original, de tal forma, que todo tipo de testimonio del pasado, siempre y cuando no interfiera en su conservación, sobreviva el máximo tiempo posible.

- Los tratamientos y materiales aplicados en conservación y restauración deben estar justificados y experimentados ampliamente en el tiempo. Nunca se debe experimentar su validez sobre un bien cultural porque cada uno de ellos es único e irrepetible. Así mismo deben ser compatibles con los originales y permitir que se realice ulteriores examen, investigaciones $y$ tratamientos.

- La discernibilidad. La intervención ha de ser fácilmente distinguible y circunscribirse a los márgenes de las pérdidas.

- Cualquier intervención ha de quedar documentada con indicación expresa del técnico que la realiza, metodología empleada, productos y proporciones utilizados en cada uno de los tratamientos efectuados.

Partiendo de estos criterios generales se han definido los criterios específicos para los bienes culturales incluidos en este proyecto en base a las necesidades demandadas por ellos 5 .

\section{Propuesta de intervención}

Una vez realizado el diagnóstico pormenorizado de estado de conservación del conjunto de bienes incluidos en este proyecto e implementado los resultados de todos los estudios preliminares, se disponen de los datos necesarios para poder definir el Proyecto de Intervención.
En esta propuesta se describirá para cada uno de los elementos constitutivos o para el conjunto, los siguientes datos:

- Tratamientos necesarios con indicación de la técnica de aplicación y de los materiales y productos a emplear.

- Cronograma de la actuación, con su respectivo faseado.

En su desarrollo se contemplarán los mismos epígrafes empleados durante la descripción del estado de conservación:

- Estructura-arquitectura:

Soporte.

Decoración polícroma.

- Elementos escultóricos:

Soporte

Decoración polícroma.

\section{Conclusión}

Como se desprende de lo expuesto en este articulo, la metodología aplicada para definir los contenidos del proyecto de intervención en el Retablo Mayor de la Capilla Real de Granada parte del conocimiento de la problemática que presenta, de sus necesidades conservativas y de la investigación técnico y científica más idónea a los fines que se persiguen, con objeto de definir la intervención más adecuada en base al diagnóstico detectado y a sus características, formales, históricas, técnicas, constructivas y conservativas. Todo ello, en consonancia con los criterios de conservación-restauración propuestos en base a las demandas de la obra, a las directrices marcadas por los documentos básicos para el desarrollo de la disciplina de la conservación-restauración de bienes culturales, y a los códigos deontológicos aceptados por los profesionales e instituciones implicados en la conservación, investigación, protección conservación y difusión del Patrimonio Histórico. 
I. Véase A.A.V.V. "Un Proyecto para la Capilla Real de Granada: Teorías, métodos y técnicas aplicadas a la conservación del patrimonio mueble". Serie Cuaderno IAPH, nº I. 1992.

2. Equipo de redacción del proyecto: ficha técnica:

Personal del IAPH:

Coordinación y diseño del Proyecto: $M^{a}$ José González López.

Mediciones de los parámetros microclimáticos y de luminotecnia y propuesta de diseño expositivo de los frontales de altar: Raniero Baglioni.

Estado de conservación y propuesta de intervención de los frontales de altar: Araceli Montero Moreno.

Estudios histórico-artístico de los frontales de altar. Gabriel Ferreras Romero.

Investigación histórico-artística del retablo: Nieves Jiménez Díaz.

Documentación fotográfica: Eugenio Fernández Ruiz.

Investigación analítica: Lourdes Martín García. Francisco Gutiérrez Montero.

Investigación biológica: Marta Sameño Puerto

Equipo de restauración:

Jefe de equipo de restauración: José María Rodríguez Acosta

Miembros del equipo de restauración: Francisco Oliver Ruiz. Amelia Cruz Guzmán. María José Ortega Gálvez. Inés Alejandra Osuna Cerdá. Ana Márquez Montoro.

Levantamiento y restitución fotogramétrica:

Map Line S.A.

Estudio de la estructura arquitectónica del reverso del retablo: Coordinación: Pedro Salmerón Escobar.

Miembros del equipo de trabajo: Elisa Entrena Núñez. María Felisa Ramírez Martín. Manuel Robles Iglesias. Susana Castro Rodríguez.
3. Técnica que permite obtener una imagen fotogramétrica a color del bien en estudio mediante el tratamiento digital de la imagen fotográfica realizada con tecnica fotogramétrica implentando los resultados de la restitución fotogramétrica.

4. Véase:

CESARE BRANDI. "Teoría de la Restauración", Alianza Forma. 1977

Ma JOSÉ MARTÍNEZ JUSTICIA. "Antología de textos sobre Restauración". Universidd de Jaén. 1996.

Guía Profesional de E.C.C.O. Actas de Ier. Congreso E.C.C.O "The conservator-Restorer's profesional activity and status and its responsability towards the cultural heritage". Florence. 1997.

5. Véase:

A.A.V.V. "Proyecto de Estudio e Intervención del Retablo Mayor de la Capilla Real de Granada" . 1998 (s.p.). 